\title{
The importance of AI for the timely detection, prevention and treatment of pandemic diseases such as COVID-19 in developing countries
}

\author{
Tahereh Javaheri $^{1}$, Khaled Seidi², Rana Jahanban-Esfahlan ${ }^{2}$, and Reza Rawassizadeh ${ }^{1}$ \\ ${ }^{1}$ Boston University \\ ${ }^{2}$ Tabriz University of Medical Sciences
}

April 28, 2020

Coronavirus disease 2019 (COVID-19), which was first reported in Wuhan in December 2019, is now widespread with outbreaks reaching across the world ${ }^{1}$. The hospital-acquired transmission rate of COVID-19 is about $41 \%^{2}$, which is due to human-to-human transmission. Compared to COVID-19, SARS $^{3}$ and MERS ${ }^{4}$ appear to have a lower morbidity rate and a higher mortality rate of $10 \%$ and $37 \%$, respectively.

Despite the high number of infections in the most developed south-eastern coastal provinces of mainland China, such as Zhejiang, they may exceed the mortality rate, as the global mortality rate of COVID-19 is about $3.4 \%^{5}$, while lower mortality rates are reported for these areas. However, no deaths are reported in Zhejiang for 1171 confirmed cases and only four deaths are confirmed in Guangdong, with 1322 true positive cases $(0.3 \%)^{6,7}$. This is a clear evidence that mortality rates are directly related to the quality of crisis management.

In contrast to all the previous outbreaks and pandemics, there is one untapped hope that could make dealing with the COVID-19 pandemic possible after all, i.e. artificial intelligence (AI) and its related technologies. For example, bioinformatics can benefit from advances in generative $\mathrm{AI}^{8}$, machine learning can assist in accurate detection of the infection ${ }^{9}$, social media analysis can raise awareness and identify cases which are not reported by governments ${ }^{10}$, simulations based on reinforcement learning ${ }^{11}$ can identify pandemic outbreak pace and magnitude and assist policy makers in their decisions.

The pandemic risk of COVID-19 results from several factors: (i) it is difficult to differentiate COVID-19 because the outbreak of the disease is associated with respiratory symptoms that are common to many other respiratory diseases such as colds and flu, which usually have a high prevalence during the cold season, (ii) COVI-19 is quite persistent and enjoys low temperatures, (iii) the transmission rate is quite high (41\%), in almost $80 \%$ of cases patients do not show typical symptoms and they could be carrier and transmit the disease to others ${ }^{12}$.

It has been estimated that there will be more deaths in developing countries, especially in countries with higher population density and lower hygienic index, and developed countries which has lots of international connections, which could contribute to an uncontrolled pandemic. Therefore, AI and its sub-branches could contribute to better management of the disease in these countries and could be beneficial in addition to human factors. Successful control of COVID-19, which has a high morbidity rate and resembles the primary symptoms of harmless respiratory diseases, depends on early diagnosis. A 14-day quarantine of false positive individuals leads to a heavy burden on health systems and ineffective disease management. Perhaps the first application of AI in a real struggle is to explore its true potential in the initial diagnosis of COVID-19 and the identification of subsequent outbreaks. 
Fortunately, the first experiment in this area was promising, in which the initial diagnosis of the disease based on a 10-15 minute computer tomography (CT) showed a diagnosis rate of $81 \%$ and with the help of AI, the detection rate could be improved up to $90 \%$ within seconds ${ }^{13}$.

What AI can potentially do to win people's battle against COVID-19 is (i) early detection of the disease with a high degree of accuracy that can save time and lead to a good decision, (ii) avoiding unnecessary quarantine of healthy people, and (iii) early diagnosis of sick individuals that can prevent further transmission of the disease. Furthermore, taking into account the demographic factors of the patient, such as sex, age and lifestyle in each country, AI can help to predict the circulation of the disease in the country and the direct use of available facilities where they are most needed. It is also possible to identify high-risk groups, related professions and companies to prevent the spread of the disease. As a point-of-care device, AI can save time, costs, energy and many lives.

AI is highly valued in developing countries, which have fewer opportunities to deal with the disease than developed countries. It can lead to the right decisions being taken at the right time and in the right place, thus providing a fair and rational distribution of opportunities to control the virus in high-risk regions. This may make developing countries less vulnerable to deaths, but given the severe economic sanctions imposed on some countries, such as Iran, many nominated companies operating in the AI sector are reluctant to do so and stand still. The fight against COVID-19 in developing countries requires full support, and in the end the whole world will benefit from it.

We also believe that it is necessary to initiate open source projects. Just a few examples show how an open source device like a ventilator can save many lives, and there are some promising efforts in this direction, such as an open source platform for surgical robotics ${ }^{14}$. The same attitude is required for algorithms and applications. At the time of writing this article, there are several preprints explaining the use of a deep learning algorithm to identify COVID-19 from pneumonia ${ }^{15,16}$ but to our knowledge, none of them have been published as open source so that other developers can use these algorithms in their application to improve their COVID-19 detection rate, which is an unnecessary investment of time and energy in reinventing the wheel in this stressed situation where time is precious and crucial.

\section{References}

1. Huang C, Wang Y, Li X, Ren L, Zhao J, Hu Y, et al. Clinical features of patients infected with 2019 novel coronavirus in wuhan, china.Lancet 2020;395(10223):497-506. doi: 10.1016/S0140-6736(20)30183-5

2. Wang D, Hu B, Hu C, Zhu F, Liu X, Zhang J, et al. Clinical characteristics of 138 hospitalized patients with 2019 novel coronavirus-infected pneumonia in wuhan, china. JAMA 2020. doi: 10.1001/jama.2020.1585 $\%$ J JAMA

3. https://www.who.int/csr/sars/country/table2004_04_21/en/.

4. https://www.who.int/emergencies/mers-cov/en/.

5. https://www.who.int/dg/speeches/detail/who-director-general-s-opening-remarks-at-the-media-briefingon-covid-19-3-march-2020.

6. Yu M, He S, Wu D, Zhu H, Webster C. Examining the multi-scalar unevenness of high-quality healthcare resources distribution in china.Int J Environ Res Public Health 2019;16(16):2813. doi: 10.3390/ijerph16162813

7. Ji Y, Ma Z, Peppelenbosch MP, Pan Q. Potential association between covid-19 mortality and health-care resource availability. Lancet Glob Health 2020:S2214-109X(20)30068-1. doi: 10.1016/S2214-109X(20)30068-1

8. Alex Z, Vladimir A, Alexander Z, Bogdan Z, Victor T, Dmitry S. B, et al. Potential covid-2019 3c-like protease inhibitors designed using generative deep learning approaches2020.

9. Song Y, Zheng S, Li L, Zhang X, Zhang X, Huang Z, et al. Deep learning enables accurate diagnosis of novel coronavirus (covid-19) with ct images. 2020:2020.02.23.20026930. doi: 10.1101/2020.02.23.20026930 $\% \mathrm{~J}$ medRxiv 
10. Gao JaZ, Pinping and Jia, Yingnan and Chen, Hao and Mao, Yimeng and Chen, Suhong and Wang, Yi and Fu, Hua and Dai, Junming, Mental Health Problems and Social Media Exposure During COVID-19 Outbreak (2/17/2020). Available at SSRN: https://ssrn.com/abstract=3541120.

11. Probert WJM, Lakkur S, Fonnesbeck CJ, Shea K, Runge MC, Tildesley MJ, et al. Context matters: Using reinforcement learning to develop human-readable, state-dependent outbreak response policies.Philosophical Transactions of the Royal Society B: Biological Sciences 2019;374(1776):1-9. doi: 10.1098/rstb.2018.0277

12. http://weekly.chinacdc.cn/en/article/id/e53946e2-c6c4-41e9-9a9b-fea8db1a8f51.

13. Sun K, Chen J, Viboud C. Early epidemiological analysis of the coronavirus disease 2019 outbreak based on crowdsourced data: A population-level observational study. The Lancet Digital Health2020. doi: https://doi.org/10.1016/S2589-7500(20)30026-1

14. Hannaford B, Rosen J, Friedman DW, King H, Roan P, Cheng L, et al. Raven-ii: An open platform for surgical robotics research. IEEE Transactions on Biomedical Engineering 2013;60(4):954-9. doi: 10.1109/TBME.2012.2228858

15. Li L, Qin L, Xu Z, Yin Y, Wang X, Kong B, et al. Artificial intelligence distinguishes covid-19 from community acquired pneumonia on chest ct.0(0):200905. doi: 10.1148/radiol.2020200905

16. Wang S, Kang B, Ma J, Zeng X, Xiao M, Guo J, et al. A deep learning algorithm using ct images to screen for corona virus disease (covid-19). 2020:2020.02.14.20023028. doi: 10.1101/2020.02.14.20023028 \%J medRxiv 